times when the circle must be contracted, to nation, family or self? I think Singer will readily agree that there are, but he has not pursued this aspect as fully as it deserves; one could start, perhaps, from the work of Coser on the functions of social conflict, or Evans Pritchard's analysis of segmentary social systems.

Second, anthropologists must surely find it hard to accept the approach to "rationality" adopted by Singer. $\mathrm{He}$ speaks of rationality as if it were a unitary phenomenon, leading properly, given a particular set of considerations, to certain conclusions. Not only is this all too rarely the case within cultures sharing one set of concepts - one language etc. - but between cultures the idea of what it is to think or behave rationally varies in many dimensions. It is doubtful indeed whether all cultures even have a notion of rationality. Who knows but it may be advantageous, in order to overcome the "cognitive dissonance" problem he refers to (p.143), to dispense with it completely?

Criticisms aside, however, this book is undoubtedly an excellent exposition of a most complex problem. Peter Singer has without question clarified the many subtleties and singularities involved in the relation between ethics and sociobiology.

Vernon Reynolds is University Lecturer in Physical Anthropology at the University of Oxford, and author of The Biology of Human Action (W.H. Freeman, 2nd Edn, 1980).

\title{
Imagination more firmly controlled
}

\section{Morris Moscovitch}

Mental Imagery and Human Memory. By J.T.E. Richardson. Pp.178. ISBN 0-33325370-1/0-312-52975-9. (Macmillan, London/St Martin’s Press: 1980.) £16, \$22.50.

AFTER being neglected for about a halfcentury by experimental psychologists in the English-speaking world, mental imagery once again become a worthy subject of inquiry in the middle 1960 s. The fact that such private, mental representations could be studied objectively signalled the end of the old stimulusresponse behaviourism and augered the birth of a new cognitive psychology that could explore freely a variety of phenomena that are part of mental life.

By the mid-1970s, however, the new cognitive psychology had become the established doctrine and research on mental imagery had grown duller while, at the same time, the controversy surrounding it had become more intense. Currently, much research on mental imagery seems to be concerned with establishing the nature of the code in which mental images are represented rather than in exploring further the nature of the phenomenon or the uses to which it can be put. One group believes that mental images are represented in a code that is fundamentally different from that in which verbal information is represented. The former is concrete, analogical and perhaps isomorphic with perception, whereas the latter is abstract and propositional. Against this dual-coding position is the view that all information is represented in terms of a common code involving networks of propositions. The controversy has taken on the trappings of an arms race. No sooner has one side unveiled a powerful empirical or theoretical weapon which threatens the other with ultimate defeat, than an equally destructive finding or argument is developed by the other side. One can only lament this expenditure of talent, because the differences between the two camps, though large in theory, have become almost indistinguishable in practice. It often takes more than an expert to decide whether an experiment struck a blow for one side or the other.

Such expert refereeing is provided by $\mathrm{J}$. T. E. Richardson in Mental Imagery and Human Memory. As the title suggests, he is concerned with mental imagery only insofar as it contributes to human memory. His aims, though limited, are not modest. They are to analyse and comment on conceptual and methodological problems and to integrate and organize the research findings in an area that has grown exponentially over the years and has become quite unwieldy as a result. By and large he has done a good job.

In the early chapters he reviews critically, yet succinctly, the major historical trends in imagery research that led to the current state of affairs. He suggests that some of the conceptual problems facing imagery research arose in part as a result of making imagery acceptable to a behaviouristically orientated psychology. By providing a conceptual analysis of mental imagery as it is used in ordinary language and psychological discourse, Richardson shows up the shortcomings of some of the current approaches and clarifies the theoretical arguments between the singleand dual-coding theorists. Particularly important here is the idea that mental images, because they are representations, have the logical feature of intentionality. That is, the object and functional origin of the image is determined by how it is intended, not by what it resembles. Thus, images arise from "a system of knowledge which is conceptual and propositional", (p.40). Once constructed, however, images may have emergent properties which could not be computed from the original descriptions and which may not be coded in propositional form.
Thus armed, Richardson reviews the experimental literature on imagery and memory. Chapters 4 and 5 are on imagery and immediate memory and on pictorial imagery, Chapters 6-8 deal primarily with imagery and long-term memory, and the last chapter concerns individual differences. He argues that whereas the effects of imagery on long-term memory for single words and sentences are best explained by a propositional theory, the use of imagery in immediate memory (such as rotating or inspecting a figure mentally) and in remembering pictures is best explained in terms of a dual-coding theory. This suggests that mental images may have different functional properties in different instances and, consequently, the evidence from these instances should be weighed separately in deciding theoretical issues.

I regret that he chose not to discuss experiments in the phenomenology of imagery (how big is an image, how wide an angle does it subtend, how long does it take to conjure it up?), since it would have fitted nicely into the chapter on imagery and working memory. I also wish that he had dealt more fully with neuropsychological approaches to problems in mental imagery. Recent investigations by Bisiach and Luzzatti (Cortex 14, 129-133; 1978), who found that individuals with left-sided neglect also neglect the left side of their mental image, suggest that such investigations have a useful contribution to make.

However, the book is an excellent and very readable source of information on research in mental imagery. Richardson has acted as referee and thereby clarified and made more interesting an area that was badly in need of organization. It is left to another book to offer new directions to research and theory

Morris Moscovitch is an Associate Professor in the Psychology Department and Centre for Research in Human Development at Erindale College, University of Toronto.

\section{Estuarine mixture}

\section{J. D. Burton}

Chemistry and Biogeochemistry of Estuaries. Edited by E. Olausson and I. Cato. Pp.452. ISBN 0-471-27679-0. (Wiley-Interscience: 1980.) £28.50, \$78.40.

THE study of estuaries presents a notable challenge to aquatic geochemists because of the range of physicochemical conditions and variations in composition encountered in estuarine waters and sediments, and the variability and complexity of estuarine environments. There have been several reasons for taking up this challenge and for the consequent emergence, during the past decade, of estuarine chemistry as a distinctive field. The complex pathways of 